\title{
Crianzas mutuas. El trato a los animales desde las concepciones de los pastores puneños (Jujuy, Argentina)
}

\author{
Lucila Bugallo \\ Instituto Interdisciplinario Tilcara (FFyL-UBA), UNIHR-UNJU, IES No 2 Tilcara (Argentina) \\ bugallolucila@yahoo.com.ar \\ Jorge Tomasi \\ CONICET, Instituto Interdisciplinario Tilcara (FFyL-UBA) (Argentina) \\ jorgetomasi@hotmail.com
}

Recibido: 1 de septiembre de 2011

Aceptado: 5 de octubre de 2011

\section{RESUMEN}

La relación entre personas y animales domésticos, específicamente en hacienda, se enmarca en el universo y prácticas pastoriles de la región andina de la Puna en la provincia de Jujuy (Argentina). La temática se aborda a partir de dos casos del área que presentan semejanzas y diferencias, ubicados respectivamente en los departamentos de Susques y Cochinoca. Las características de esta economía pastoril se presentan como un marco de contextualización, para abordar después los espacios de los animales, en particular los corrales, y su comparación con las casas de la gente, así como la inclusión de la hacienda en tanto que 'pariente'. Se muestra cómo el vínculo entre personas y animales está atravesado por una concepción de la reproducción de la hacienda en la que incide, entre otros factores, la suerte del criador. Este vínculo crea una afinidad entre las personas y ciertos animales, promoviendo una crianza en la que el cariño tiene un lugar central.

Palabras clave: Sur andino, prácticas pastoriles, espacios de los animales domésticos, parentesco, crianza

\section{Mutual Raising. The Treatment of Animals from the Views of the Puna Herders (Jujuy, Argentina)}

\begin{abstract}
The relationship between people and domestic animals is framed within the pastoral universe and practices in this Andean area at the highlands in the province of Jujuy (Argentina). The thematic is approached from two cases that present similarities and differences in the departments of Susques and Cochinoca. The characteristics of this pastoral economy is presented as a framework for contextualization, then the areas of animals are described and discussed, including corrals and a comparison with the houses [casas] of people, and the inclusion of the animals as relatives. It shows how the link between people and animals is crossed by a conception of the reproduction of the herd, which is affected, among other things, by the luck [suerte] of the breeder. This link creates an affinity between people and certain animals, promoting a breeding where affection is essential.
\end{abstract}

Key words: South Andes, pastoral practices, spaces of animals, kinship, breeding

Sumario: 1. Introducción. 2. Aproximación a las prácticas pastoriles en la puna. 3. Los espacios de la hacienda. Los múltiples corrales de los pastores puneños. 4. La hacienda como parte de la familia. 5. Consideraciones finales. Cariño y crianza. 6. Referencias bibliográficas. 


\section{Introducción}

Este trabajo se propone abordar la relación entre las personas y los animales domésticos en el área de la Puna en la provincia de Jujuy, Argentina, dentro de los Andes meridionales. Las relaciones entre animales y personas están en esta zona, como en la región andina en general, enmarcadas en una concepción de la naturaleza, del espacio, de las plantas e igualmente de los animales, que no permite una objetivación de los otros seres en tanto recursos, insumos, materiales u objetos. Por el contrario, se considera la co-existencia e interrelación de una amplia gama de seres que interactúan desde sus propios lugares y perspectivas, algunos revistiendo en tanto $w^{\prime} k^{\prime} a^{1}$ un carácter sagrado, siendo considerados seres tutelares o divinidades, como en el caso de los cerros, ojos de agua, ciertas piedras, etc.

Es en este marco en el que se sitúan las relaciones entre animales y personas y es así como, por ejemplo, una pareja de novios-ovejas puede tener padrinos y madrinas humanos, los animales de las tropas tienen casas, coquean y el día de su señalada entran, junto con sus parientes humanos, en diálogo con la pachamama como entidad genérica del trato y diálogo con la tierra, pastos y agua. Al ser considerados de esta manera, los animales domésticos son tratados como parientes, con cariño y afecto, se los conoce individualmente, con sus mañas y virtudes.

El trabajo se refiere a la relación con los animales de las tropas -rebaños de camélidos-, dejando de lado otro tipo de animales domésticos. Cabe aclarar que la gente puneña no distingue a los animales domésticos en oposición a los salvajes, sino que estas categorías se definen por las relaciones y prácticas que se establecen con unos y otros. La relación con los animales de las tropas se sitúa en el ámbito de las prácticas pastoriles, incidiendo éstas en la totalidad del modo de percibir el entorno, el espacio y la diversidad de especies tanto animales como vegetales.

Es importante señalar que nos referiremos a dos áreas de la Puna jujeña, Susques y Cochinoca que, a pesar de situarse ambas en el altiplano, presentan varias diferencias empezando por las características ecológicas que condicionan la posibilidad de ciertos tipos de producción ${ }^{2}$. Por lo tanto, si bien la población a la que nos referimos es de tipo pastoril, no lo es en los dos casos de estudio de la misma manera: en Susques su economía se basó durante gran parte de su historia en el pastoreo de diferentes especies; en Cochinoca, la cría de distintas especies de ganado, se combinó con una agricultura de autoconsumo en pequeña escala. A su vez, en ambos casos las economías domésticas se transformaron profundamente durante el siglo XX, por la inserción de los puneños en el mercado de trabajo asalariado.

Nos proponemos, por lo tanto, desplegar una pequeña parte de este mundo de relaciones, de cariño y consideración entre personas y animales de la tropa en esta región andina, abordando tanto sus diferencias y semejanzas en lo que hace a las prácticas productivas, como en lo que se refiere a un modo de concebir y tratar a los animales.

1 Aclaramos que la utilización de bastardilla para subrayar algunos términos, indica que éstos son vocablos en otro idioma o que, si se trata de palabras en castellano, éstas tienen un uso específico/local en este contexto.

2 A lo largo del trabajo sólo se especificará Susques y/o Cochinoca en el caso de que se trate de una referencia propia de una de las dos áreas; por el contrario, si no se indica es porque lo que se refiere es válido para ambas. 


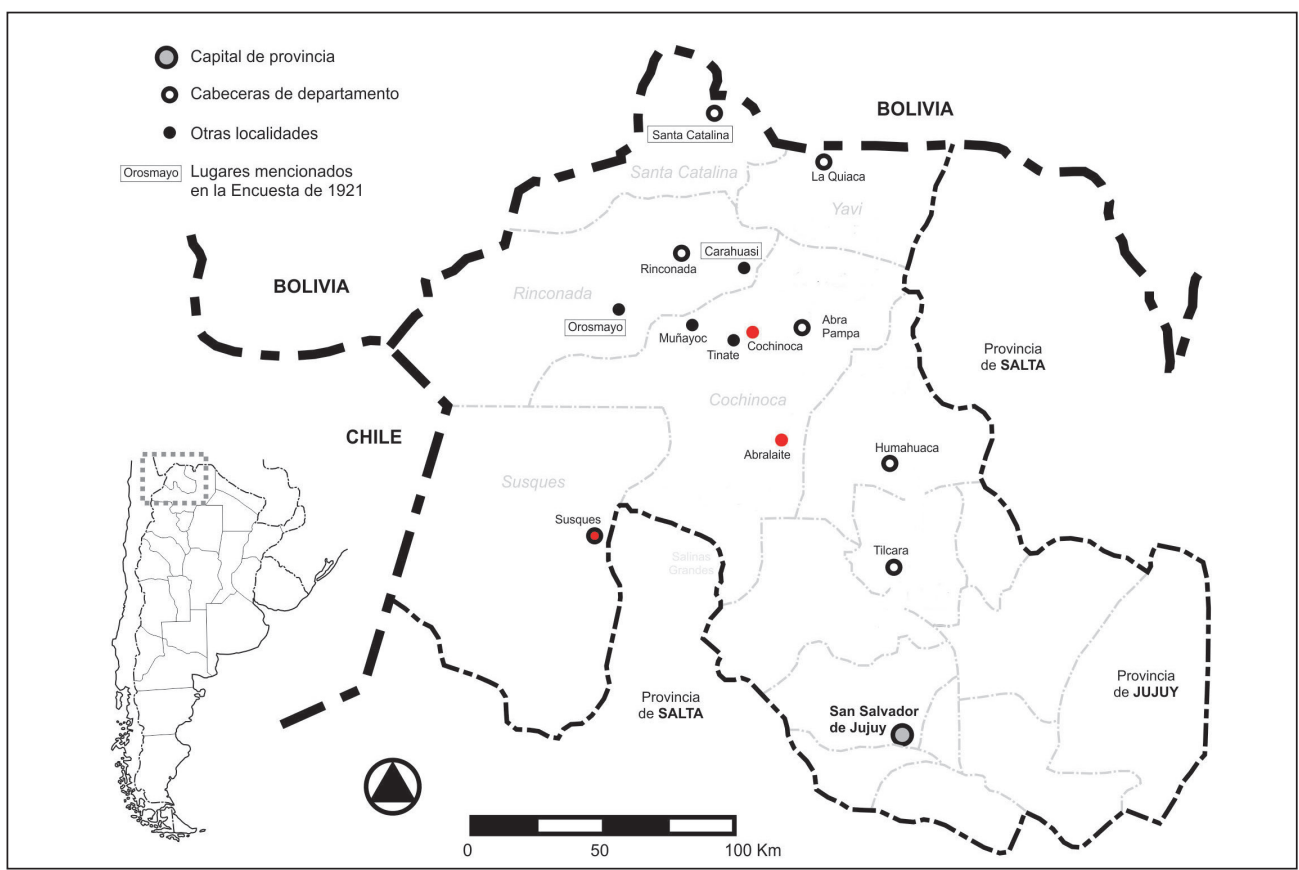

Figura 1: Mapa de la provincia de Jujuy con el detalle de los lugares mencionados en el texto.

Consideramos que resulta interesante abordar el tema de la relación entre personas y animales en la Puna jujeña, comparando y contrastando dos sub-áreas, ya que a la vez que se presentan datos etnográficos de una zona poco estudiada y con escasa presencia en los estudios andinos en general, se hace mostrando a la vez su diversidad. Es decir, estos Andes del altiplano jujeño contienen una complejidad y no representan una región homogénea.

Las fuentes con las que hemos trabajado son mayormente etnográficas, resultado de trabajos de campo llevados a cabo en ambas áreas puneñas. En el área rural circundante al pueblo de Susques se ha venido desarrollando trabajo etnográfico desde 2004, en el marco de una investigación sobre las espacialidades pastoriles (J. Tomasi); otros diferentes lugares del área centro oriental puneña (departamento de Cochinoca), principalmente el área de Abralaite, han sido igualmente objeto de estudio etnográfico desde 1998 (L. Bugallo). Se han incorporado además otras fuentes, como los testimonios de la Encuesta Nacional de Folklore de 1921, con los cuales se han excedido los límites de las áreas puneñas antes referidas debido al interés que presentan estas descripciones (Figura 1).

El texto comienza con una contextualización que nos introduce en las prácticas pastoriles de esta área andina, para adentrarse luego en las tres cuestiones centrales que pretende abordar: los espacios del ganado, los animales como parientes y el cariño con que son tratados en su crianza. 


\section{Aproximación a las prácticas pastoriles en la puna}

Los vínculos entre las personas y los animales en la puna se dan en el marco particular que presentan las prácticas y relaciones dentro de las dinámicas propias del pastoreo. Distintas etnografías han mostrado las especificidades que asume esta actividad a lo largo de los Andes, en un primer momento para Perú, y luego para grupos pastoriles de Bolivia, Chile y las tierras altas del norte argentino (p.e. Abeledo 2008; Göbel 2000/02; Merlino y Rabey 1978; Tomasi 2011; Yacobaccio et al. 1998).

El pastoreo extensivo es un tipo de actividad económica orientada a la cría de ciertas especies animales que es particularmente viable en algunos ambientes. En la definición del pastoreo dada por Núñez y Dillehay (1995 [1979]:155) para el contexto andino, éste es considerado como un sistema económico de producción, en el que se da una interacción entre seres humanos y animales que es única, por estar centrada en un seguimiento transhumante de los movimientos cíclicos de los animales que depende de la estacionalidad y de la conducta móvil de los mismos. Esta movilidad estacional y cotidiana establece una estrecha relación entre animales y personas; resulta fundamental observar que estos desplazamientos conjuntos nos muestran no sólo una cotidianeidad sino también una existencia compartida e interdependiente.

Dentro de un universo de prácticas comunes entre los grupos pastoriles en los Andes, los casos que estamos trabajando (Cochinoca y Susques) nos permiten observar las variabilidades significativas que existen en la intensidad de la movilidad, la cantidad de asentamientos o la conformación de los rebaños. Es necesario aclarar la terminología utilizada por los puneños para referirse a sus animales. La hacienda es la totalidad de animales que tiene una familia, en cambio la tropa es de cada especie, por ejemplo la tropa de llamas. Ganado remite al vínculo con el estado, ya que se refiere a los ganados en el caso de censos y algunos puneños lo vinculan con los animales vacunos; para ellos las llamas no serían ganado.

La unidad doméstica se constituye, al igual que en otros lugares, como el núcleo central de producción y reproducción en el contexto de estos grupos pastoriles (Khazanov 1994). Estas unidades domésticas se componen de personas que, en la mayoría de los casos, tienen lazos de parentesco consanguíneo y de afinidad entre sí e ingresan a grandes rasgos dentro de lo que localmente se conoce como «familia».

En Susques el pastoreo, aunque ya no constituya la principal fuente de ingresos, sigue siendo una actividad importante y la mayor parte de las unidades domésticas siguen sosteniendo rebaños de diferentes dimensiones. En los últimos años se ha consolidado el trabajo asalariado, al que se incorporaron las poblaciones puneñas a principios del siglo XX, y la mayor parte de los miembros de las unidades domésticas tienen empleos públicos o privados en el pueblo y en otros centros urbanos. Es decir que hoy en día los que están al cuidado de la tropa en el campo suelen ser una media de dos o tres personas e incluso hasta una sola; el resto de los miembros suelen tener animales dentro del rebaño familiar y participan de algunas actividades (señalada, arreglo de corrales, carnear).

En muchas unidades domésticas de la zona de Cochinoca las familias han dejado de criar ovejas y cabras y han privilegiado la cría de llamas que necesitan de menos cuidados. Incluso varias han alambrado y van cada semana a ver las llamas 
encerradas en estos lotes cercados, un cambio significativo para las prácticas de pastoreo tradicionales. Don Ciriaco Condorí de Tinate, nos dice «no tenemos ninguna cabra, las llamas único, las cabras s'ha acabado todo, vacas también había, todo s'ha acabado» ${ }^{3}$. Sin embargo, en términos sociales y simbólicos, el pastoreo y la cría de animales siguen teniendo un lugar central para estas poblaciones. Aunque ya no sea una práctica cotidiana, se constituye como un mundo de representaciones heredadas, que conforman el modo de pensar y de vincularse con los animales. Es interesante que cuando un pastor o pastora deciden no dedicarse ya a la cría, lo expresan en términos de «acabar», como don Ciriaco que expresa todo s'ha acabado. No parece hablar solamente de una majada, o de un recurso económico, es un modo de vivir y relacionarse con el mundo que se acaba con cada tropa que se va. Pero los puneños de más edad llevan este «mundo» consigo, y por eso sigue constituyendo un universo de significado presente y potente.

Dentro de una división del trabajo por género, las mujeres están a cargo del cuidado y responsabilidad cotidiana de los rebaños. Son las pastoras las que se encargan de cuidar a la tropa en los recorridos para pastear en el día a día, por lo que tienen un rol fundamental en el diseño de las estrategias de movilidad estacional. Son ellas quienes mejor conocen a los animales y sus necesidades y éstos las reconocen a ellas. Los hombres se ocupan de otras tareas, como el manejo de pequeños cultivos, la construcción y reparación de los corrales o casas, o ciertas actividades anuales (castraciones, esquileos, etc.). Eran los hombres quienes realizaban los viajes de intercambio a otras zonas ecológicas, vinculando a la unidad doméstica con el «mundo de afuera». Esta división del trabajo se ha sido observada en ambas áreas de estudio, así como por otros investigadores en la región de la Puna jujeña. (Bratosevich 1992; Göbel 1998; Merlino y Rabey 1983). Debido a lo señalado sobre la organización del trabajo en el seno de las unidades domésticas, actualmente tal división ya no está tan vigente, especialmente en la zona de Abralaite.

Una modalidad habitual para conservar una tropa sin dedicarse cotidianamente al pastoreo es entregarla a una persona ajena a la unidad doméstica. El trato puede ser «al cuidado» $\mathrm{o}$ «al partir»; ambas son formas mediante las cuales una persona puede entregar una cantidad de animales a un pastor/a para que la maneje. Es común que el pastor contratado lleve sus propios animales a pastar junto con la tropa que le es entregada. En el caso de un trato «al partir», las crías o multiplicos serán divididos entre el dueño y el pastor/cuidador. En cambio «al cuidado» las crías son del dueño pero el pastor/a es retribuido por su trabajo.

Es importante que nos detengamos en la conformación de los rebaños. Al igual que en otros puntos de los Andes, tanto en Susques como en Abralaite los criadores suelen tener distintas especies: llamas, ovejas y cabras, e incluso en algunos casos vacas. Esta diversificación es importante en relación con las estrategias de pastoreo, ya que cada una tiene ciertos requerimientos, preferencias y temperamentos que imprimen particularidades al manejo, los recorridos y el uso de los asentamientos a lo largo del

\footnotetext{
3 Conversación con don Ciriaco Condorí, doña Panta Mamani de Condori y doña Eduarda Cruz. Tinate (departamento de Cochinoca), 20/02/2011. Don Ciriaco Condorí nació en Tinate en 1920, tiene 90 años; actualmente reside en San Salvador de Jujuy (la ciudad capital), junto a su señora Panta, su hija y nietos.
} 
año ${ }^{4}$. Algunos autores han marcado que el reparto de especies constituye una estrategia para el manejo y reducción del riesgo (Cfr. Browman 1990; Göbel 1997).

Por otra parte, las tropas están compuestas por animales de diversos dueños, generalmente miembros de la unidad doméstica, salvo cuando los animales son dados, como hemos indicado, al partir. Sin embargo, aunque los animales tengan un dueño individual, se trata de rebaños familiares. Los miembros de la familia reciben animales en suerte, principalmente cuando son niños, para la ceremonia del primer corte de cabello o simplemente se les nombra ${ }^{5}$ durante la señalada. De alguna manera, los niños se crían en una historia compartida con los animales que recibieron en suerte y crecen juntos. Cuando una mujer forma una familia, suele llevarse los animales que le pertenecen y así inicia su propia tropa. Otra posibilidad es que los padres, ya mayores, entreguen a sus hijos e hijas el ganado que a cada uno le corresponde. El compartir el rebaño mediante los tratos al partir o al cuidado es una forma específica de construir relaciones fuera del grupo e incorporar personas a las redes de colaboración. Lo que nos importa señalar en esta ocasión es que a través de los animales se crean vínculos entre las personas.

La señal en la oreja y las flores y chimpos de lana que se colocan durante la señalada, son los mismos en toda la tropa, remiten a su identidad y su vínculo con ese grupo familiar, y no son propios de cada dueño individual. De hecho los colores para el ganado se heredan, generalmente de madre a hija, por lo que marcan la relación de esa tropa con un linaje. Linaje humano y linaje de la hacienda se relacionan íntimamente mediante los colores. Los pastores y pastoras reconocen, sin embargo, a cada uno de los animales porque «son dueño, lo conocen todo, yo por ejemplo lo conocía tooodo [con énfasis], quien de quien, quien de quien, cual es de quien, aquel corderito de quien es, todo, cuando vivía ahí» (entrevista con Orlando Tinte ${ }^{6}, 02 / 12 / 2008$ ).

Las unidades domésticas pastoriles controlan un determinado territorio de pasturas, los pastoreos, que implican derechos y transferencias intergeneracionales. Es así que el espacio se fragmenta y cada parte o porción se asocia con una familia, generándose un vínculo estrecho entre familia y pastoreo. Dentro de estos pastoreos cada familia tiene una cierta cantidad de asentamientos propios: una casa principal en el campo, que en Susques se conoce como domicilio, y un promedio de unas cuatro o cinco estancias o puestos en los cerros, en el caso de Susques, y uno o dos para Abralaite. Estas estancias suelen encontrarse a una distancia que van desde los quince minutos hasta una jornada de caminata desde la casa principal. La ubicación de estas estancias surge de una compleja trama de factores que incluye las necesidades emocionales de la hacienda y la apropiación de lugares significativos. En términos generales, podríamos observar que las estancias están insertas de un modo estricto en el mundo de la crianza y manejo de la hacienda en el día a día, con lo que se relacionan sus configuraciones y emplazamientos (Tomasi 2011).

4 En Susques un rebaño promedio tenía en el 2009 alrededor de 126 animales con un máximo de 472 y un mínimo de 12 . Del total de animales un $42,72 \%$ eran cabras, el $28,7 \%$ eran llamas, el $28,11 \%$ eran ovejas y finalmente las vacas sólo representaban un $0,47 \%$ del total (Censo Ganadero 2009, Comunidad Aborigen «Pórtico de los Andes», Susques).

$5 \mathrm{Al}$ acto de entregar un animal se le dice nombrar: por ejemplo «a Rita le han nombrado una cabra».

${ }_{6}$ Orlando Tinte es originario de Muñayoc, nacido en 1981, y residente en la quebrada de Humahuaca. 
A lo largo del año los miembros de la unidad doméstica junto con el rebaño, se desplazan entre estos asentamientos siguiendo un cierto ciclo que es flexible pero tiende a repetirse en el tiempo. En el caso de Susques la permanencia en cualquiera de los asentamientos oscila entre los quince días y los tres meses. De esta manera se presenta una notable intensidad de desplazamientos en comparación con otros grupos pastoriles descritos en los Andes, de un modo tal que puede implicar incluso hasta más de diez cambios de asentamiento por año. Aunque pudiendo resultar un tanto esquemático, podríamos sintetizar este conjunto de asentamientos como un ciclo circular entre el campo y los cerros. La mayor parte de las unidades domésticas suelen estar en el domicilio durante al menos parte de los meses de lluvia, entre diciembre y marzo, para luego comenzar los recorridos por las estancias de los cerros en los meses de seca $^{7}$. La decisión de trasladarse a una determinada estancia no surge exclusivamente de una decisión estratégica unilateral de las personas, sino que se consideran también las preferencias de la hacienda. Se dice que, al igual que las personas, los animales tienen gustos y deseos que tienen que ser considerados por la pastora. Ese es el pasto de ellos es una expresión muy común en la puna para referir que ese pasto les gusta a esos animales en cuestión, que comen ese pasto. Al decir es de ellos, se refiere a que ese pasto les gusta pero que además les pertenece; los animales de la hacienda mantienen una relación profunda con sus pasturas.

\section{Los espacios de la hacienda. Los múltiples corrales de los pastores puneños}

A partir de su trabajo en Qaqachaka, en el altiplano boliviano, Arnold (1998: 36) ha propuesto que la casa «se convierte en una representación del cosmos, una metáfora del cerro mundo, un axis mundi, y una estructura organizativa en torno a la cual giran otras estructuras». En el mismo sentido, Nielsen (2001: 43) observó que la casa «puede ser analizada como 'cosmograma' que sintetiza el entendimiento práctico del mundo que distingue a cada cultura». Tanto en la organización de la casa como en su proceso de construcción se ponen en juego y actúan aspectos fundamentales de la sociedad, de modo que no es posible considerarla como un mero contenedor pasivo de prácticas.

Una cuestión por demás importante, que han señalado también distintos autores, es que existe un paralelo entre la configuración de la casa y la organización de los grupos domésticos, de forma tal que ambos estarían atravesados por los mismos principios ordenadores (Cfr. Arnold 1998; Palacios Ríos 1990). A través del modo en que los distintos recintos se organizan en torno a un patio, se están evidenciando las relaciones de parentesco y la pertenencia de una persona a una determinada línea de descendencia. En este sentido, la casa podría ser pensada como una cartografía de las

7 Esta movilidad estacional existía años atrás igualmente en el área de Abralaite. Actualmente, como la mayoría de las unidades domésticas redujeron o acabaron sus tropas, extinguiéndose casi las ovejas y cabras, las estancias del cerro han sido en la mayoría de los casos abandonadas. Se utilizan las pasturas vinculadas a la casa principal del pueblo, en la ladera de sierra y las de la pampa de la laguna, ambas situadas en la zona llamada campo. 
relaciones entre las personas ${ }^{8}$, entendiendo que la casa no es simplemente una consecuencia de los lazos que existen, sino que participa en su constitución. Las relaciones que se materializan en la casa no se limitan a las personas, sino que involucran también a los animales dando cuenta de los vínculos que existen y del rol que les cabe a éstos dentro de la conformación de la familia. Los corrales son parte de la casa, tanto como los animales son miembros del grupo doméstico. En la casa se espacializan y materializan las relaciones entre personas y animales, a través de las articulaciones entre los lugares de unos y otros.

En Susques como en Cochinoca, la casa principal en el campo, se conforma a partir de la suma de una cierta cantidad de recintos techados que se despliegan en torno a un patio que tiende a estar delimitado en sus lados. La casa principal es una construcción absolutamente dinámica que se va transformando con la construcción de nuevos recintos, los que se suman como resultado de los cambios que ocurren a lo largo del desarrollo del grupo doméstico (Tomasi 2011). En este sentido, la casa tiene una cierta vida y, al igual que la familia, se podría decir que se va criando y creciendo. Cada uno de estos recintos que componen la casa puede tener funciones específicas, a veces superpuestas, como espacios para dormir, ramadas, cocinas cubiertas, depósitos, oratorios, etc. Más allá de los usos que tengan cada uno de estos recintos, se les denomina casa, al igual que al conjunto general de recintos. Es decir la casa se compone de casas. La noción de casa hace referencia, entonces, tanto al todo como a la parte, entendiendo que esa parte se constituye también como una totalidad en sí misma (Tomasi 2011).

Siguiendo el mismo ordenamiento conceptual, los corrales son las casas de la hacienda. Esto es central para comprender las relaciones entre personas y animales a través de sus espacios. Aprehender que los corrales son casas tanto como las de las personas, nos aproxima a una condición de los animales que no se presenta desde una oposición personas-animales, sino más bien desde existencias compartidas e interdependientes. Los corrales tienen particularidades constructivas y de configuración que los diferencia de las casas de las personas, pero no son ontológicamente diferentes. Podemos partir de una primera dimensión de análisis observando que ambos son espacios delimitados, cerrados y contenidos en sus lados por pircas. Es decir, se trata de espacios recortados, connotados y significados de modos particulares. Tal como propuso Delfino (2001), el pircar no es sólo una necesidad de demarcación de propiedad, sino que se trata de una práctica de significación y creación de un límite de sentido.

La concepción de corral presente en estas zonas, no se remite únicamente al recinto que permite encerrar a la tropa para protegerla y para evitar su dispersión, sino que refiere asimismo a un modo de pensar, lo que es encerrado y de este modo consolidado en tanto unidad. Este consolidarse a partir de un espacio, que implica dentro/fuera, interior/exterior, se vincula a su vez con la noción de suerte, central en el pensamiento económico puneño. El corral es junto con las pasturas el espacio y ámbito de la hacienda, pero es en el corral donde la potencialidad de reproducción o multiplico de la tropa se despliega y donde debe cuidarse y permanecer. La tropa, a pesar de

8 Con el mismo criterio Arnold se refirió a la casa en términos de una «arquitectónica de fundamentos andinos de parentesco y descendencia» (1998: 38). 
estar constituida por animales individuales, reconocidos como tales por sus dueños, se considera en ciertos aspectos como una unidad. Esta unidad es la que comparte la suerte, la que tiene ánimu, y es esta disposición anímica la que debe permanecer en el corral, debe ser encerrada y no tiene que escapar, para lo que se deben tener ciertos cuidados.

Existen otras relaciones significativas entre los corrales y los espacios de las personas. Dentro de la casa, la cocina exterior se conoce como fueguero, kancha o kanchero, y es éste uno de los espacios más importantes de la cotidianeidad de la familia, ya que en torno al fogón se cocina y se reúnen las personas. Los corrales y kancheros son espacios que no están techados y ambos están cerrados por muros bajos de alrededor de 1,5 metros de alto construidos en pirca seca, es decir con piedras que están unidas entre sí por forma y no por el uso de algún tipo de argamasa. El uso del término quechua kancha para referirse al fueguero, nos aproxima aún más a las relaciones entre los espacios de las personas y los de la hacienda. Este vocablo suele designar a espacios abiertos rodeados por una pirca. González Holguín (1608) en su vocabulario indica que la cancha es «el patio o corral», «corral de ganado» se diría llamacancha, mientras que «patio de casa» sería cancha. En otras zonas andina es común «referirse al patio de la casa como un corral de animales» (Yapita y Arnold 1998: 210). Entonces tanto animales como personas tienen su kancha: espacios abiertos aunque contenidos.

Las casas de las personas como las de la hacienda presentan sus entradas hacia el este ${ }^{9}$. Esta preferencia no puede reducirse simplemente a la necesidad de aprovechamiento de la radiación solar por la mañana, especialmente si consideramos que el corral es un espacio destechado. La dirección este o naciente, está vinculada con el sol y con lo fecundo, germinante y vivo, en cambio el oeste o poniente indica la dirección de la región de los muertos ${ }^{10}$.

En el centro de las casas de las personas se encuentra la boca de la pachamama, pachero en el área de Cochinoca y juiri en Susques, y lo propio ocurre en el centro del corral. Esa boca es alimentada en momentos muy específicos que involucran a las personas y a los animales: durante el mes de agosto, en los pacheros de los patios, cuando se da de comer a la pachamama, y en los corrales en las señaladas del verano en los corrales. A pocos centímetros de la boca/pachero del corral suele haber una piedra mediana o grande donde, durante la señalada, se coloca la coba con la que se sahuma a la tropa y a las personas, y que se constituye como marca del lugar de la boca a lo largo del año ${ }^{11}$.

9 En el caso de las casas de las personas el este es la orientación preferencial aunque no la única. La configuración en torno a un patio como organizador que busca cerrarse por todos sus lados implica que no siempre es posible disponer las puertas hacia el naciente. En todo caso, la primera casa siempre se abre en esa dirección. Cuando se trata de los corrales, la orientación sí es siempre hacia el este. Las tumbas de los muertos también suelen estar orientadas hacia el este.

10 Muchas prácticas puneñas se realizan mirando hacia el naciente: cuando se hacen ofrendas y chayas (ch'allas), cuando se flora y marca la hacienda, cuando se sacrifica un animal, éste debe mirar hacia el este.

11 En un corral en Susques se presentaba la particularidad de que se había dejado una hornacina en el muro en un punto diametralmente opuesto a la entrada que se utilizaba con ese fin. Por su ubicación remitía a las hornacinas que se dejan en el muro posterior de los oratorios donde se colocan los santitos. 


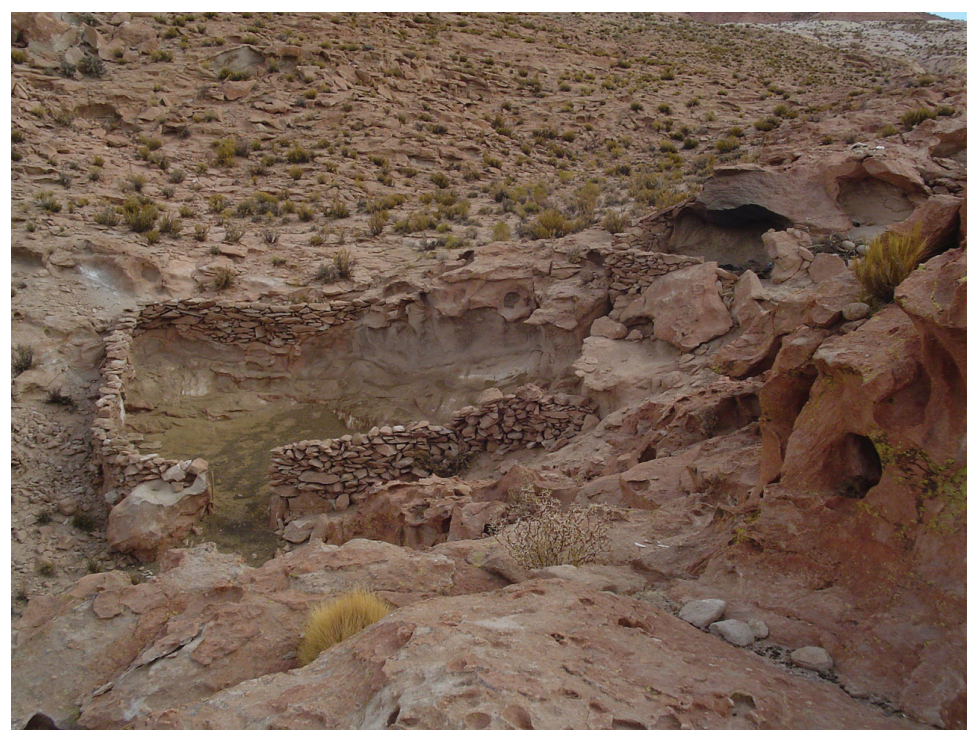

Figura 2: Fotografía del corral (abajo) y el fueguero (arriba) en una estancia en los alrededores de Susques.

Los corrales suelen estar próximos a la casa para que la pastora pueda escuchar durante la noche si ocurre algo con la tropa. En general los domicilios tienen entre uno y tres corrales dependiendo de la cantidad de hacienda que tenga la familia. En muchos casos, dado que en las estancias los recintos para las personas se limitan a un fueguero exterior, la incidencia de los corrales es mucho más grande. Podríamos decir que los corrales son el centro privilegiado de las estancias y constituyen las estructuras que reciben la mayor dedicación constructiva (Figura 2).

A diferencia de lo que ocurre con las casas de las personas, no suelen construirse nuevos corrales, sino que se mantienen y arreglan los realizados años atrás por los abuelos ${ }^{12}$. En general suelen ser circulares o rectangulares, aunque los primeros son mucho más comunes ${ }^{13}$; en algunos casos se utiliza la técnica de construcción con «champas» ${ }^{14}$ de guano cortadas directamente del piso del corral en lugar de la pirca seca, que también es utilizada sólo en parte para elevar la altura algunos centímetros por encima del muro de piedra. Con el mismo objetivo es bastante común ver ramas de arbustos que se colocan paradas a modo de última hilada.

12 En la puna jujeña se utiliza abuelo y abuela, o los abuelos, como un término general de respeto, por lo tanto no implica siempre una relación de filiación/descendencia.

13 Esto debería relativizarse en el caso de las estancias donde la morfología de los corrales está en relación con el aprovechamiento de los accidentes topográficos, como peñas, aleros o pequeñas quebradas. Además algunos corrales tienen en el costado una pequeña estructura similar, de no más de un metro de diámetro, el chiquero, que es el corral de las crías más pequeñas.

${ }_{14} \mathrm{La}$ «champa» es una técnica de construcción con tierra bastante extendida en ciertas regiones aunque no es tan común en la Puna. A diferencia del adobe que es moldeado, las «champas» son bloques que se cortan directamente en el suelo, aprovechando las raíces de la vegetación que consolidan la tierra. En los alrededores de Susques hemos registrado una única casa, en este caso de personas, construida enteramente con «champas» de guano. 


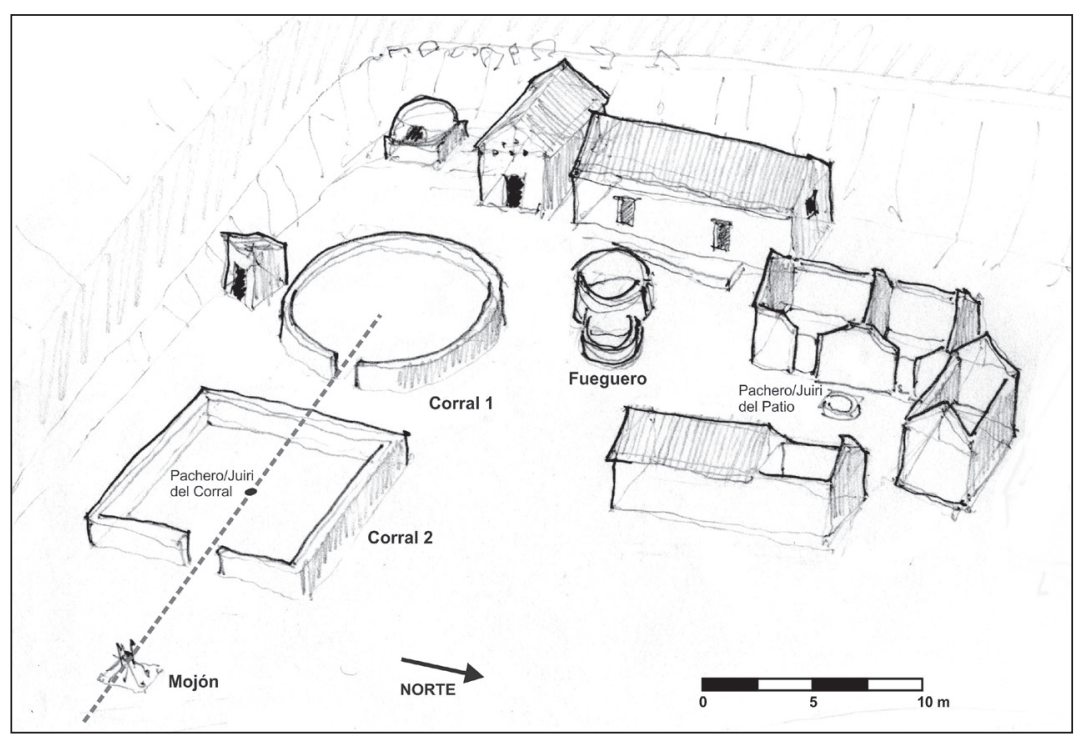

Figura 3: Detalle del mojón, «encerrado en su corral» en una casa principal.

La entrada al corral, como dijimos, está siempre construida hacia el este. Los corrales de la casa principal tienen, a una decena de metros de la entrada y alineado con ésta, el mojón del ganado. En ciertas áreas puneñas las familias poseen dos mojones: uno de las llamas y otro para las demás especies criadas ${ }^{15}$. Los mojones son pequeñas estructuras de forma cónica de algo menos de un metro de alto, construidas con piedras superpuestas de un modo similar al de las apachetas. La relación entre el mojón, la entrada al corral y la boca de la pachamama en el interior, conforman un eje en sentido este-oeste que divide en mitades el corral, pero a su vez marca un sentido de recorrido. Por su ubicación, cuando la tropa sale del corral necesariamente lo hace hacia el naciente y debe pasar por el mojón antes de salir a pastear; lo mismo ocurre con la caída del sol, cuando la tropa regresa al corral mirando hacia el poniente, necesariamente tiene que atravesar el mojón. En este contexto el mojón remite a la idea de apacheta, no sólo por su forma, sino también por su sentido como marcador de camino ${ }^{16}$. A su vez, el mojón podría asociarse con un límite, como el punto donde termina el espacio de la casa (Figura 3).

En el momento de la señalada el mojón se constituye como un núcleo central del ritual en el que se condensan las relaciones entre las personas y los animales y la totalidad del espacio de la familia. Este ritual se cierra con una puesta en acto de la compra-venta de ganado: los participantes llevan piedras que juntan en el campo, las que el dueño o dueña de la tropa compra con coca. Éstas son chayadas y floradas con lana de chimpar, lana kunti-lana en vellón teñida generalmente en la escala del rojo,

\footnotetext{
15 Los mojones dobles están presentes en todos los corrales del área de Santa Catalina, y también los hemos podido ver en Tinate, departamento de Cochinoca.

16 En una señalada de Tinate hemos visto que los dueños de la tropa indicaban que ésta, una vez señalada y florada, debía pasar en medio de ambos mojones.
} 
de carácter ritual- al igual que los animales de la tropa y son, finalmente, colocadas en el mojón, que se convierte en una pequeña montaña de piedras que se van acumulando año tras año. Entonces se confecciona un pequeño corral: con las ramas de tola a modo de postes y la lana kunti entrecruzada como los hilos del alambre, se arma el corral del mojón ${ }^{17}$. Una vez concluida esta tarea, los dueños encierran su ganado, chayando las piedras y arrojándoles coca (Bugallo 2011). Los mojones son entonces considerados como un conjunto de animales vivos encerrados en su corral (Figura 4).

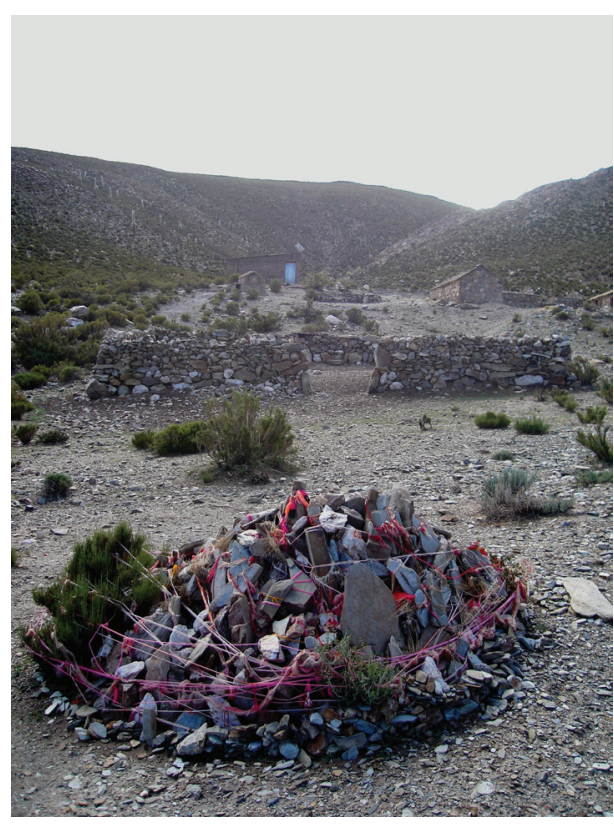

Figura 4: Esquema de una casa principal en Susques con la alineación del mojón, el pachero y la entrada al corral.

En relación con la concepción de la suerte y de lo que debe ser encerrado en el corral, que mencionábamos más arriba, es interesante observar que el día de la señalada no se permite a nadie entrar al corral sin cubrirse la espalda, con poncho los hombres y manta las mujeres; de lo contrario se dice que se «está pelado» (desnudo). Un testimonio de 1921 explica que «de esta manera queda la suerte en la casa» ${ }^{18}$. Estar pelado es, entre otras cosas ser pobre, no tener hacienda; se trataría de un criador que ha perdido $s u$ suerte. Muchas prácticas puneñas se sustentan en la idea de mantener la suerte en la casa, en el corral. El testimonio citado refiere que actuando de tal modo, la suerte queda en la casa; ya que la prescripción refiere al recinto o espacio del corral, vemos que el corral se vuelve un equivalente de «casa». La suerte del dueño de la tropa, que es la suerte de la casa y que, además, vincula a las personas con los animales como veremos más adelante, debe ser siempre cuidada. En ocasión de la

\footnotetext{
17 Estos corrales de los mojones los hemos visto en el área de Yavi, y también construidos junto a la gente durante las señaladas en el área de Cochinoca; en cambio en Susques no se hacen estos pequeños corrales.

18 INAPL. Archivo de la Encuesta Nacional de Folklore 1921, Caja 3, carpeta No 52, Casabindo fj.18 r. En adelante ENF 1921
} 
venta de animales o antes de carnearlos, se les saca un pequeño mechón de lana que se guarda: «Es la suerte del dueño, como el animal ya se va, para que no se la lleve, para que no pierda la suerte» (conversación con Teresa Condori, originaria de Muñayoc, 24/08/2009). Lo mismo cuenta un testimonio de Carahuasi: «Antes de vender un cordero o llama, le sacan un poco de lana del lado izquierdo, serca del corazón que le llaman (guaca de la hacienda) la guardan bien porque es la suerte» (ENF 1921, Caja 2, carpeta $\mathrm{N}^{\circ} 35$, Carahuasi, fj $12 \mathrm{v}$ ). Y uno de Orosmayo explica que estos mechones se guardan junto a los pedacitos de oreja en una chuspa ${ }^{19}$, para luego entregárselos a la pachamama en el centro del corral con coca y alcohol, «para que haya igual multiplico, se satisfaga la Pacha Mama (madre de la Tierra) y no se heche de menos entre sí la hacienda. Pués cuando así no se hace, pena la hacienda y se muere» (ENF 1921, Caja 3, carpeta $\mathrm{N}^{\circ}$ 26, Orosmayo, fj $10 \mathrm{r}$ ). Los mechones de lana, profundamente vinculados con la suerte, quedan en el corral o en chuspas, que en ocasiones también permanecen en el corral (Bugallo 2009).

Pero al ser colocados en chuspas son igualmente encerrados, ya que las chuspas son también consideradas como corrales. Las chuspas, confeccionadas con la lana de los animales, contienen la suerte de la tropa, sus mechones y lanas, sus pedacitos de oreja, la coca que ellos pastan. En ellas viven las illas o wak'as de la tropa-pequeñas figuras de piedra en forma de animal-, según dice un puneño, las chuspas son corrales, en los que los animales-illa pastan su pasto de coca; en ciertas fechas, como señaladas y pascua, se saca a estos animalitos de su «corral tejido» y se les renueva el pasto, colocando nueva coca. Las chuspas presentan este aspecto de continente que las vuelve corral, como espacio cerrado que encierra cierta energía vital. También los animales poseen sus chuspas: en las señaladas se les colocan a ciertos animales en el costado una bolita de lana kunti, algodón y coca, enredándola en su propia lana; en su chuspa el animal «lleva guardado todo, lleva guardada toda su riqueza» (conversación con Teresa Condori, 24/08/2009) (Bugallo 2011).

Los puneños y puneñas, como la mayor parte de los andinos, leen diferentes señas para conocer el estado de las cosas: su suerte, el clima, el tomar decisiones, etc. Para las tistinchas ${ }^{20}$ de San Santiago el 25 de julio y de pachamama en agosto, se colocan entre las mazorcas que se hacen hervir, algunas a las que se les han quitado ciertos granos. En éstas se leerá luego la suerte de la tropa, su multiplico, si «hay suerte, las ovejas van a llenar el corral, van a multiplicar, llenito va estar el corral como se llenó la mazorca» (conversación con Orlando Tinte, 08/2005). Existen distintas prácticas rituales y cotidianas destinadas a valorar y potenciar «lo lleno», aunque éste no es el lugar para desarrollarlas. Quisiéramos de todos modos llamar la atención sobre el hecho de que los corrales, los mojones de la hacienda y las chuspas, son pensados de esta manera, como espacios que deben encerrar lo lleno, en el sentido de completo, de rebosante. Los dos corrales en miniatura, el del mojón y la chuspa, son elaboraciones que encierran la concepción puneña de la fertilidad y la reproducción de las tropas. La relación entre linajes familiares y corrales es expresada por la gente de Qaqachaka

\footnotetext{
19 La chuspa es una pequeña bolsa tejida en lana con una tirita para colgarla del cuello, siempre en colores y con algún diseño textil, en donde tradicionalmente se guardaba la coca.

20 La comida ritual llamada tistinchada se prepara con ingredientes secos -mazorcas, habas, carnes con hueso como cabezas- que se hacen hervir en grandes ollas al fuego durante toda la noche.
} 
vinculada con la matriz materna: la barriga de la que, como de un corral, salen los hermanos; éstos son un rebaño que sale de ese vientre-corral (Yapita y Arnold 1998: 207).

\section{La hacienda como parte de la familia}

«Uno tiene un cariño inmenso por sus animales, ¿por qué crees que cuidan y todo?, parte es como una familia porque vos casi que te preocupas lo mismo que te preocupas por tu familia por tu hacienda. Porque hay gente que no quiere dejar su hacienda, que no lo quiere acabar» (conversación con Orlando Tinte, 08/2011).

Esta manera de concebir a la hacienda como parte de la familia, toma un matiz particular el día de la señalada de los animales de la tropa, ya que en ciertas partes de la puna jujeña se establece un parentesco ritual entre animales y personas, convirtiéndose estos últimos en padrinos y madrinas de los novios de la tropa (Figura 5). Así lo realiza doña Dionisia Zulca con sus llamas y ovejas. Al comienzo de la señalada se elige la pareja de novios; debe ser un casal de orejanos, lo que significa que no deben haber sido marcados anteriormente. A los novios se les eligen padrinos entre las personas presentes y, entonces, se lleva a cabo el casamiento. Éstos, ubicados mirando hacia el este, la hembra del lado izquierdo, son bañados por una lluvia de mistura (talco y papel picado) y quedan así blanqueados; se los junta, haciendo un lazo de serpentinas alrededor de sus cogotes. Una vez casados, se les da de tomar chuya con coca y se les cortan las orejas, imprimiéndoles la señal del dueño/a. Se les pone flores en sus orejas y chimpos o tulmas en sus lomos y, por ser los novios, llevan más chimpos que cualquier otro. Con la sangre del corte de oreja se marca en forma de cruz la mejilla derecha o la frente de los participantes ${ }^{21}$ (Bugallo 2011). En Muñayoc también se eligen padrinos y madrinas humanos.

Juan Alfonso Carrizo había observado esta práctica a principios del siglo XX. Escribe que los dueños de la tropa nombran a los padrinos de la ceremonia, aclarando que «el compadrazgo recae en las personas distinguidas de la concurrencia, en los dueños de rebaños más numerosos (...). Tomados del brazo como si estuvieran en un casamiento, van los padrinos seguidos del dueño de casa y de toda la concurrencia al corral en donde bala el ganado» (Carrizo 1959 [1934]: XLVIII) ${ }^{22}$. En su descripción, los padrinos se hacen cargo de la ceremonia, lo que se diferencia de lo que hemos conocido actualmente, ya que en nuestro caso hemos visto a los dueños de la tropa realizar las señales y abrir el pachero en el centro del corral.

En cambio don Zenón, de Abralaite, recuerda cómo era allí antes, entonces los padrinos eran animales como también ocurre actualmente en Susques. "Yo hago mi parte como sabía hacer finada mi madre, diferente, como dicen bien florado, los chicos [los animales jóvenes] tenía que elegir para el padrino, la madrina, otro para el ahi-

21 Esta descripción se basa en las señaladas de doña Dionisia Zulca, en Abralaite, entre los años 1999 y 2004.

22 Si bien la edición original es de 1934, sabemos que Carrizo realizó su trabajo en la puna anteriormente; por ejemplo estuvo en Cochinoca hacia 1925. Aunque Carrizo no especifica a qué lugar refiere la señalada que describe, por otras indicaciones conocemos que participó de señaladas en Rinconada y en el pueblo de Cochinoca. 
jado, para la ahijada. El padrino con la ahijada, la madrina con el ahijado, tapas con unas mantas para que limpie todo, purifica todo y salen todos, todos bien enflorados», agrega «ese es el estilo de años» (entrevista con don Zenón Piñero, 24/02/1999) ${ }^{23}$.

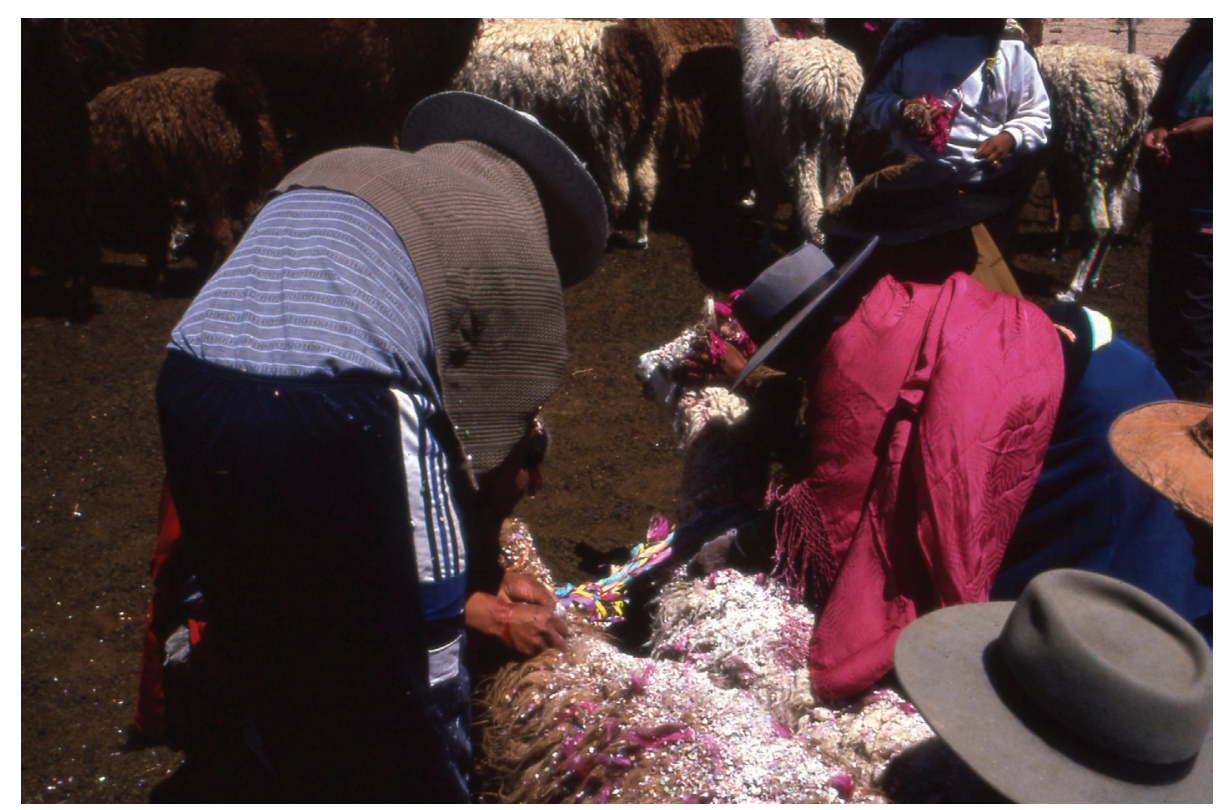

Figura 5: Casamiento de los novios de una tropa de llamas durante la señalada, en Abralaite.

Es interesante lo que señala Carrizo, ya que este compadrazgo posibilita formalizar una relación entre personas de diferentes estatus económico-social. Como escribe Spedding (1998: 133) «El compadrazgo permite la formación de lazos duraderos entre personas de diferentes clases sociales y comunidades de origen que nunca podrían convertirse en afines». Podríamos pensar que esta característica del compadrazgo se puede extender a las relaciones en las que los ahijados son animales. Asimismo debemos considerar que se establece igualmente un lazo entre gente y animales, o entre humanos y no-humanos, en el caso de los novios-animales de algunas señaladas del área de Cochinoca que tienen padrinos humanos, así como en el compadrazgo que se da entre los dueños de la tropa y los padrinos animales de las señaladas de Susques. Se trata de establecer lazos, no sólo entre personas de diferente pertenencia socioeconómica, sino entre diferentes clases de seres, mediante el único tipo de parentesco posible entre ellos. En Susques, donde los padrinos son animales adultos de la tropa, éstos deben presentar rasgos ejemplares en cuanto al temperamento, la capacidad reproductiva, los colores, etc.; el añacho (el macho hechor) suele ser el padrino. Vemos que esta «regla» sigue la norma general, según la cual los padrinos de matrimonio deben constituir un ejemplo en tanto pareja, ser una pareja ejemplar «Esto porque los

23 Nacido en Abralaite en 1938. La entrevista tuvo lugar en Abralaite (departamento de Cochinoca). 
padrinos deben guiar a los ahijados, aconsejarles, e intervenir cuando tengan problemas» (Spedding 1998: 125). En las señaladas, los padrinos y los dueños de la pareja de novios, es decir de la tropa, se convierten en compadres. Los padrinos deben participar en la señalada, intercediendo y propiciando el multiplico de la tropa a la que sus ahijados representan. Vemos además que los novios son como hijos para los dueños de la tropa.

Las personas y los animales se vinculan a través de una relación que es en cierto modo propiciatoria; hay personas que tienen suerte para tal animal y otras que no, éstas pueden tener suerte para otro tipo de animal, la suerte debe probarse y de ese modo se descubre. Parecería existir algo así como una «afinidad» con esa especie animal que no se da con otras, una relación particular entre esa persona y esos animales, y es ésta la que propicia la fecundidad y multiplico de la hacienda (Bugallo 2009). El hecho de que la suerte de una persona se vea en el multiplico de una especie de animales, muestra que las suertes de ambos están vinculadas: ese animal se reproduce por el vínculo con su dueño/a y a la vez esa persona tiene la posibilidad de subsistir gracias al animal. ¿Cómo es este vínculo? ¿Cómo se establece? El acto de entregar el primer animal con el que comienza o no la suerte de la persona con esa especie, se designa nombrar. Es el nombrar lo que implica propiedad y pertenencia, ya que éstas no se marcan en el cuerpo del animal (como dijimos la señal es una sola para toda la tropa). Entonces el vínculo entre la persona, su suerte y el animal se da a través del nombre: el nombre de la persona dueña individual de ese animal, al que le dará su suerte para su multiplico. Félix Palacios (1988: 149) escribe que cuando alguien no tiene suerte con los animales es porque los espíritus de éstos no le han adoptado. La relación se explicaría ya sea por una entidad vinculada con los animales criados que tiene una afinidad con esa persona, o por la suerte de la persona que beneficia al animal. De una u otra forma, se refiere a una energía vital que permite la reproducción y la regeneración de la vida y que se despliega a través de la relación persona-animal (Bugallo 2009).

La manera de vivenciar la relación entre animales criados y gente parece estar atravesada por un fuerte hilo que los une; así durante las señaladas las personas deben coquear (mascar coca) para que haya pasto para la hacienda «los abuelos, [para que] no falte ni agua ni pasto para la hacienda, coqueaban los abuelos» nos cuenta don Ciriaco Condori ${ }^{24}$. Lo mismo dice doña Dionisia Zulca: «Coqueen, coqueen para que hayga pasto» (señalada de doña Dionisia Zulca, Abralaite, 23/02/2006). Como si las personas «rumiaran» la coca, y en ese salivar y degustar fueran criando pasturas para sus animales.

\section{Consideraciones finales. Cariño y crianza}

Podemos decir que todas estas relaciones con los animales de las tropas, considerando a sus corrales como casas y a ellos como parientes, se enmarcan en un vínculo de cariño, concebido como un criar y ser criado. Como lo expresan los pastores peruanos

24 Conversación con don Ciriaco Condorí et al. Ver nota 3. 20/02/2011. 
de Chichillapi «nosotros criamos al ganado y el ganado nos cría a nosotros» (Palacios Ríos 1977: 58).

A los animales se les trata con cariño, como las personas, tienen nombres, se les habla; en otras zona andinas se les canta una diversidad de canciones según las épocas del calendario (Arnold y Yapita 1998). Algunos nombres se refieren a sus edades; así don Zenón les dice a sus llamas chicos, tekesitos cuando son pequeños, y cuando son recién nacidos panzonas, cuando crecen son maltonas; las llamas que no recrían son las solteras. También las nombra según su sexo, les dice chinas y changos: "Changuito le digo a los chicos a todos les digo changuito. Las chinitas, que son chinitas son hembritas, digo 'son chinitas ustedes y vos te llamás chango, nada y vos te llamas china' (...) ellos entienden, los changuitos también entienden, los chiquitos también entienden» (entrevista con don Zenón Piñero, Abralaite, 24/03/1999). Hay modos relacionados con el contexto ritual y con el cariño, así las llamas son pairumas para la abuela Panta y doña Francisca, dos abuelas de Cochinoca. Algunos animales tienen sus nombres propios, en general relacionados con el color de su lana, y suelen ser nombres de aves: se llaman pato, parina, guayata (ganso andino), gaviota, suri ${ }^{25}$. «Gaviota, gaviotita, frente blanquita, le digo larguita porque es larguita aquí, tiene su frentecita blanquita. De ahí le he puesto larguita, de esa forma. Por ahí le pongo nombre así, porque francamente como suri son peliadores (...) ya me entienden, ya le hablo así, ya me entienden, le grito, se dan la vuelta y se vienen» (entrevista con don Zenón Piñero, Abralaite, 24/02/1999). Pero también se las reta, doña Damiana se enoja con sus ovejas cuando no hacen caso, don Zenón les dice marrajas a sus llamas cuando no vuelven «no vienen, no vienen se tiran en el campo y no van a venir».

Sin embargo, la noción de crianza incluiría a todos los seres y no sólo a los animales: «una crianza en un mundo vivo» en que el agua, el clima y todos los seres y entidades son criados. Así «el mundo andino es un mundo de crianza en el que la sabiduría consiste en saber criar y en saber dejarse criar» (Kessel y Enriquez Salas 2002: 60-61). Los animales son considerados como seres que poseen intencionalidad y capacidad de vincularse e interactuar con una diversidad de otros seres presentes en el espacio de la vida. Así, ellos pagan su pasto y establecen intercambios directos con pachamama; cuando los animales de la tropa pierden sus flores en el campo la gente dice que «hacen cambio con pasto». Don Zenón recordaba lo que le decía su madre «el pasto que ellos comen, con la lana pagan ellos, dejan chimpito, chimpito cuando van al campo dejan por ahí, con eso pagan ellos para que coman su pasto» (conversación con doña Dionisia Zulca, entrevista con don Zenón Piñero, 24/02/1999). Lo que implica una concepción que ubica a los animales en ese «espacio vivo», interactuando y alimentando con chimpos a la pacha. Es decir criándola y dejándose criar con el pasto.

Así, con nombres, buenos tratos, enojos, en el conocimiento mutuo, se van tejiendo las relaciones con los animales de la hacienda. Como con los familiares, en la cotidianeidad del pastorear, en los corrales. Criándose mutuamente.

25 Este modo de nombrar a la hacienda está presente en toda la región andina, especialmente en lo que refiere a las llamas (Flores Ochoa 1978; Palacios Ríos 1977). En los contextos rituales de las señaladas las llamas son chullumpi (Kessel y Enríquez Salas 2002: 71), siendo chullumpi un ave acuática. 


\section{Referencias bibliográficas}

Abeledo, Sebastián

2008 Los Pastores de la Altura. Una mirada evolutiva de la vida en la Puna de Atacama. Tesis de licenciatura en Ciencias Antropológicas. Facultad de Filosofía y Letras, Universidad de Buenos Aires.

ARnOLD, Denise

1998 «La casa de adobe y piedras del Inka: Género, memoria y cosmos en Qaqachaka», en Hacia un orden andino de las cosas, Denise Arnold, Domingo Jiménez y Juan de Dios Yapita, eds., pp. 31-108. La Paz: Hisbol/ILCA.

Arnold, Denise y Juan de Dios YAPITA

1998 Río de vellón, río de canto. Cantar a los animales, una poética andina de la creación. La Paz: ILCA/Hisbol

BRATOSEVICH, Nicolás

1992 «Estructura agraria en la región de la Puna. Casabindo 1986-1987», en Sociedady articulación en las tierras altas jujeñas. Crisis terminal de un modelo de desarrollo, Alejandro Isla, comp., pp.115-165. Buenos Aires: Ecira-Asal-MLAL.

BRowman, David

1990 «High altitude camelid pastoralism of the Andes», en The World of Pastoralism. Herding Systems in Comparative Perspective, John Galaty y Douglas L. Johnson, eds., pp. 323-352. Nueva York: The Guilford Press.

Bugallo, Lucila

2009 «La noción de 'suerte' en el centro de la relación ser humano-mundo», en Congreso Nacional y Surandino de Filosofía, San Salvador de Jujuy, (ms).

2011 «Flores para el ganado. Una concepción puneña del multiplico (puna de Jujuy, Argentina)», en Las herranzas en los Andes, Juan J. Rivera Andía, comp. En prensa.

CArrizo, Juan Alfonso

1959 Cancionero popular de Jujuy [1934]. Gobierno de la Provincia de Jujuy, Universidad Nacional del Tucumán.

Delfino, Daniel

2001 «Las pircas y los límites de una sociedad. Etnoarqueología en la Puna (Laguna Blanca, Catamarca, Argentina)», en Ethnoarchaeology of Andean South America, Lawrence A. Kuznar, ed., pp. 97-137. Ann Arbor: International Monographs in Prehistory.

Flores OCHOA, Jorge

1978 «Classification et dénomination des camélidés sud-américains». Annales. Économies, Sociétés, Civilisations 5-6: 1006-1016.

GöBEL, Bárbara

1997 «'You Have to Exploit Luck': Pastoral Household Economy and Cultural Handling of Risk and Uncertainty in the Andean Highlands». Nomadic People 1 (1): 37-53.

1998 «Salir de viaje’: Producción pastoril e intercambio económico en el noroeste argentino», en 50 años de estudios americanistas en la Universidad de Bonn: Nuevas contribuciones a la arqueología, etnohistoria, etnolingüistica y etnografía de las Américas, Sabine Dedenbach-Salazar et al., eds., pp. 867-891. Markt Schwaben: Anton Saurwein. 
2000/02 «Identidades sociales y medio ambiente: la multiplicidad de los significados del espacio en la Puna de Atacama». Cuadernos del Instituto de Antropología y Pensamiento Latinoamericano 19: 267-296.

2002 «La arquitectura del pastoreo: Uso del espacio y sistema de asentamientos en la Puna de Atacama (Susques)». Estudios Atacameños 23: 53-76.

González Holguín, Diego

1608 Vocabulario de la lengua general de todo el Peru llamada lengua Qquichua, o del Inca. Lima.

Kessel, Juan van y Porfirio EnRiQuez Salas

2002 Señas y señaleros de la madre tierra. Agronomía andina. Quito: Abya Yala / IECTA.

Khazanov, Anatoly

1994 Nomads and the Outside World. Madison: The University of Wisconsin Press.

MerLino, Rodolfo y Mario RabeY

1978 «El ciclo agrario-ritual en la puna argentina». Relaciones de la Sociedad Argentina de Antropología 12: 47-70.

1983 «Pastores del altiplano meridional: religiosidad, territorio y equilibrio ecológico», Allpanchis 21: 149-171.

NiELSEN, Axel

2001 «Evolución del espacio doméstico en el norte de Lípez (Potosí, Bolivia): CA. 9001700 DC». Estudios Atacameños 21: 41-62.

NuÑEz, Lautaro y Tom DilLeHAY

1995 Movilidad giratoria, armonía social y desarrollo en los Andes Meridionales: Patrones de tráfico e interacción económica [1979]. Antofagasta: Universidad Católica del Norte.

Palacios Ríos, Félix

1977 «'...Hiwasaha uywa uywataña, uka uywaha hiwasaru uyusitu'. Los pastores aymara de Chichillapi». Tesis de Maestría. Lima: PUCP.

1988 «Pastores de llamas y alpacas», en Raíces de América: El mundo aymara, Xavier Albó, comp., pp. 133-151. Madrid: Alianza Editorial.

1990 «El simbolismo de la casa de los pastores Aymara», en Trabajos presentados al simposio «rur 6. El pastoreo altoandino: origen, desarrollo y situación actual», Jorge Flores Ochoa, ed., pp. 63-83. Cuzco.

Sppeding, Alison

1998 «Contra-afinidad: algunos comentarios sobre el compadrazgo andino», en Gente de carne y hueso. Las tramas de parentesco en los Andes, Denise Y. Arnold, comp., pp.115-137. La Paz: CIASE/ILCA.

Tomasi, Jorge

2011 Geografías del pastoreo. Territorios, movilidades y espacio doméstico en Susques (provincia de Jujuy). Tesis de doctorado en Geografía, Facultad de Filosofía y Letras, Universidad de Buenos Aires.

Yacobaccio, Hugo, Celina M. Madero y Marcela P. Malmierca

1998 Etnoarqueología de pastores surandinos. Buenos Aires: Grupo de Zooarqueología de Camélidos. 
YAPITA Juan de Dios y Denise Y. ARnOLD

1988 «Lo humano y lo no humano en Qaqachaka: categorías aymaras de parentesco y afinidad», en Gente de carne y hueso. Las tramas de parentesco en los Andes, Denise Y. Arnold, comp., pp.199-225. La Paz: CIASE/ILCA. 